\title{
Primary Data Treatment Software for Position-Sensitive Detector of Small-Angle Neutron Scattering Spectrometer in the Isotropic Pattern Scattering Case
}

\author{
Alexei Soloviev ${ }^{1, \star}$, Sergei Kutuzov ${ }^{2, \star \star}$, Olexander Ivankov ${ }^{3, \star \star \star}$, and Alexander Kuklin ${ }^{3, \star \star \star \star}$ \\ ${ }^{1}$ Laboratory of Information Technologies, Joint Institute for Nuclear Research, Dubna, Russia \\ ${ }^{2}$ Dzhelepov Laboratory of Nuclear Problems, Joint Institute for Nuclear Research, Dubna, Russia \\ ${ }^{3}$ Frank Laboratory of Neutron Physics, Joint Institute for Nuclear Research, Dubna, Russia
}

\begin{abstract}
A new data converter has been created for the new position-sensitive detector (PSD) of small-angle neutron scattering (SANS) spectrometer YuMO. In the isotropic pattern scattering case, it provides the possibility for processing PSD data with the SAS data processing program that has already been in use.
\end{abstract}

\section{Introduction}

The modern trends in use of the small-angle neutron scattering (SANS) method consist in varying the sample environment, e.g., temperature, pressure, magnetic or electric fields [1-5]. The objects under investigation can be oriented in the field or by the flow of a liquid. In this case the need for the position-sensitive detectors (PSD) arises.

A PSD of a new type has been created for the YuMO spectrometer. Currently, this spectrometer has a two-detector system with coaxial ring detectors with a central hole $[6,7]$ and lacking the azimutal angle sensitivity. The SAS package [8-10] is used for its data treatment. The PSD is planned to be mounted either in the place of one of the YuMO detectors, or in addition to them (turning it into a three-detector system). The PSD data processing is of the utmost importance.

In the present paper we discuss a technique of data processing which significantly improves the statistics of the PSD dependent data and makes possible the use of the allready existing on-line data processing package SAS.

\section{Current state of primary data treatment for YuMO spectrometer}

The key element of the modernized time-of-flight SANS YuMO spectrometer is its two-detector system. It provides a unique dynamic range of the neutron momentum transfer $\left(Q_{\max } / Q_{\min }\right.$ is about 90$)$ and asks for short data acquisition times. The main view of the setup of the two-detector modernized YuMO spectrometer as well as an instantiation of a SANS experiment graphic user interface (GUI)

\footnotetext{
$\star$ e-mail: solovjev@jinr.ru

$\star \star$ e-mail: kutuzov@jinr.ru

$\star \star \star$ e-mail: aleksandr.ivankov@gmail.com

$\star \star \star \star$ e-mail: alexander.iv.kuklin@gmail.com
} 
are presented in figure 1. For a detailed description of the operating mode of the spectrometer in the two-detector variant, see $[6,7]$.

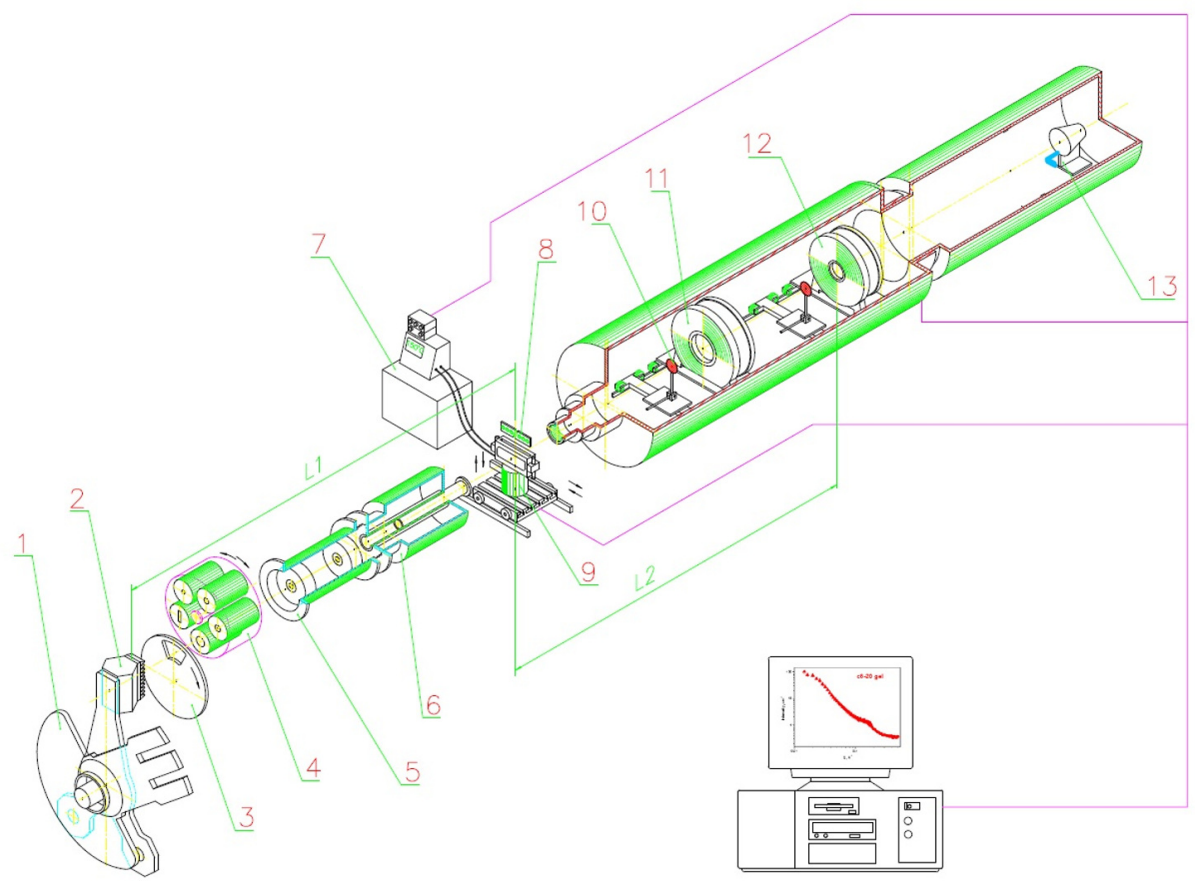

Figure 1. The setup for small-angle scattering of neutrons YuMO (4-th channel IBR-2): 1 - reflectors, 2 active zone of reactor with moderator, 3 - chopper, 4 - changeable collimator, 5 - vacuum tube with additional background collimators, 6 - justified collimator, 7 - thermostat, 8 - holder with samples on the table with thermos boxes, 9 - table of samples, 10 - the vanadium standard, 11, 12 - detectors ("OLD" and "NEW" accordingly), 13 - direct beam detector.

\subsection{SAS package}

The SAS package [8-10] is used to process the spectra measured on the YuMO spectrometer. In general, processing with SAS includes two main stages:

1. normalization of the time spectra over the scattering from the vanadium standard;

2. conversion of the time spectra into the neutron momentum transfer space.

SAS does not operate with the "OLD" and "NEW" detectors separately, and does not sew spectra from them. Instead, it operates with a set of their rings and produces the final spectrum in a natural way [9].

The main SAS window is shown in figure 2. One can see that the statistics at the end of the time spectra is poor. It negatively impacts on the normalization procedure and has to been improved. Within SAS, the combination of the neighbouring cells of the time histograms is used to this purpose.

\section{Primary data treatment for PSD}

The PSD of a new type has been created and tested for the YuMO spectrometer [11]. Typical patterns of scattering are shown in figure 3. For most of the experiments one needs the isotropic pattern of scattering only. 


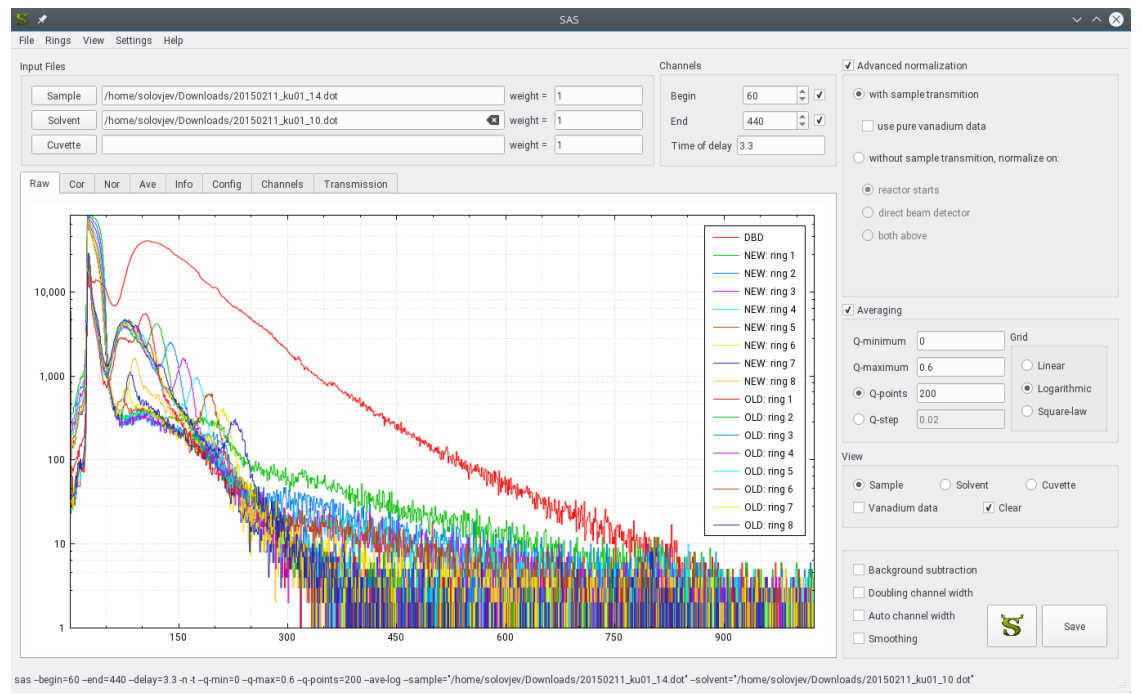

Figure 2. Main SAS window. Time spectra on detector rings.

Due to the small size of the PSD cells the statistics collected on them is often poor. That fact negatively impacts on the data treatment. On the other hand, in most of the experiments one needs an isotropic pattern of the scattering only, when objects in the sample are not oriented. In this case, it is possible to combine the PSD cells into concentric rings characterized by a single data value. This has two desireable features: statistics improvement and straightforward applicability of the on-line SAS package implementation for the data processing.
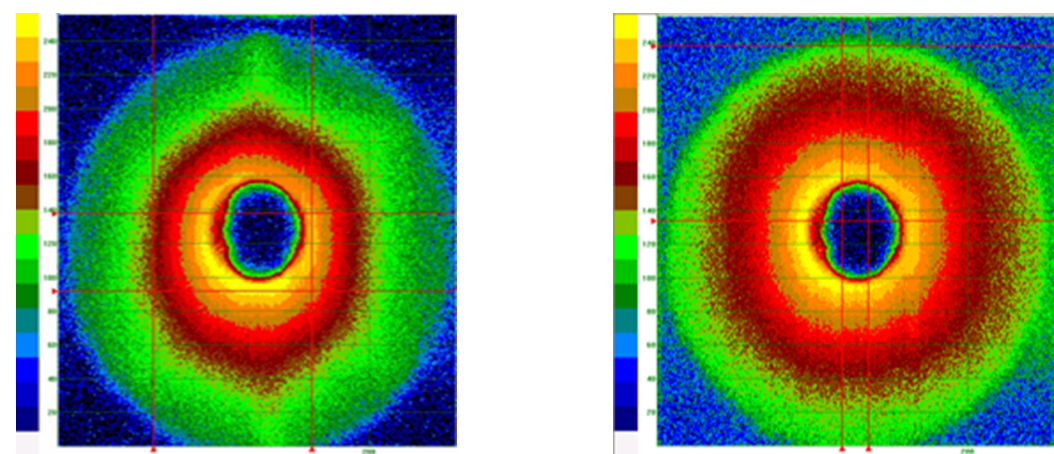

Figure 3. Anisotropic (left) and isotropic (right) patterns of scattering.

\subsection{PSD2SAS data converter}

The data converter for PSD has been created [12]. It is implemented as ROOT-based [13] Qt Application. The intersection area of a cell with the resulting ring is taken into account. The entire area of the detector is in use. OpenMP parallelism is used both for reading data and for ring construction. 

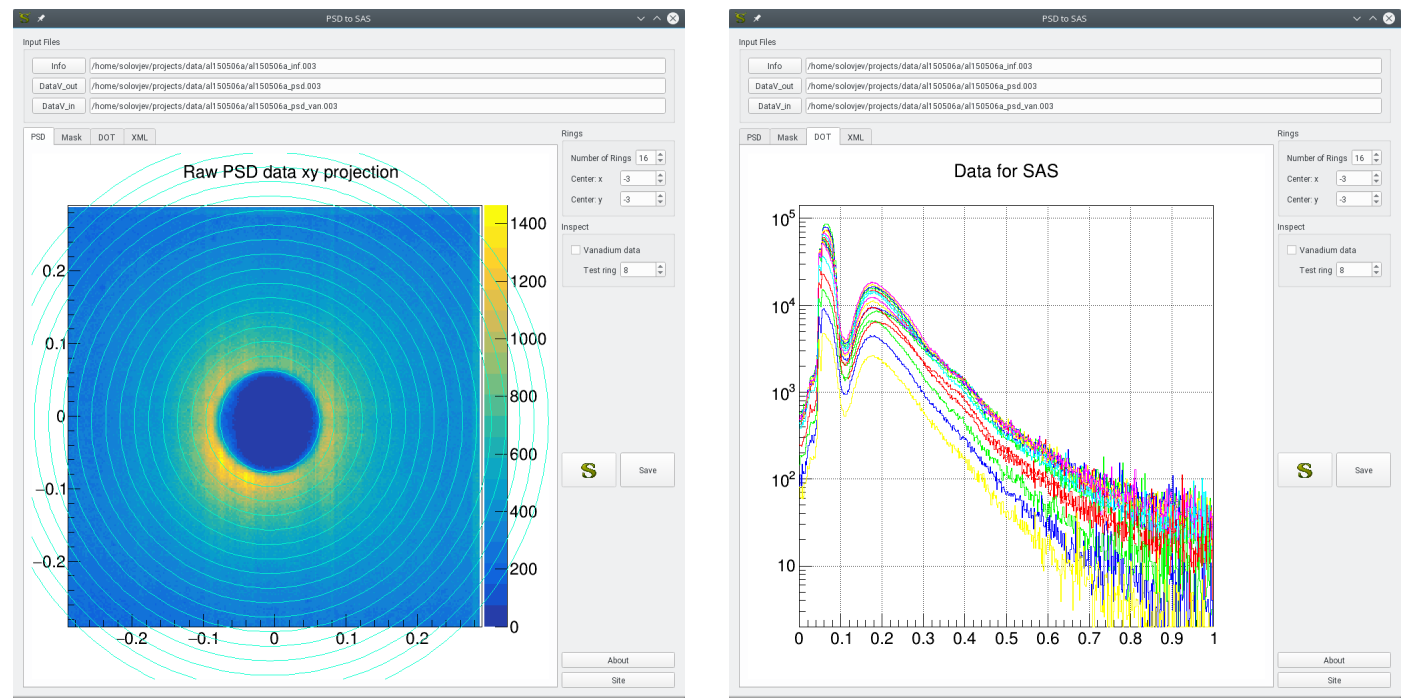

Figure 4. PSD rings pattern (left) and time spectra (right) for these rings, ready for processing in SAS

\section{Conclusion}

- The data converter for position-sensitive detector has been created.

- The $Q$-resolution provided by the detector is practically fully available.

- The $Q$-range provided by the detector is not truncated due to the use of detector edges.

- The converter is fast, the time is comparable to the processing time in SAS.

\section{References}

[1] D.V. Soloviov et al., Journal of Physics: Conference Series 351, 012010 (2012)

[2] D.V. Solov'ev et al., Journal of Surface Investigation. X-ray, Synchrotron and Neutron Techniques 5, 7 (2011)

[3] H. Gibhardt, C. Haramagatti, A. Islamov, O. Ivankov, A. Ivanovich Kuklin, G. Eckold, 228, 769 (2014)

[4] M. Balasoiu, A. Kuklin, 61 (2016)

[5] H. Gibhardt, C. Haramagatti, A. Islamov, O. Ivankov, A. I. Kuklin, G. Eckold (2015)

[6] A.I. Kuklin, A.K. Islamov, V.I. Gordeliy, Neutron News 16, 16 (2005)

[7] A.I. Kuklin et al., Journal of Physics: Conference Series 291, 012013 (2011)

[8] A.G.Soloviev, T.M.Solovieva, A.V.Stadnik, O.I.Ivankov, A.I.Kuklin, Communication of JINR P10-2003-86 (2003)

[9] A.G. Soloviev, T.M. Solovjeva, O.I. Ivankov, D.V. Soloviov, A.V. Rogachev, A.I. Kuklin, Journal of Physics: Conference Series 848, 012020 (2017)

[10] https://wwwinfo.jinr.ru/programs/jinrlib/sas/indexe.html

[11] A.I.Kuklin et al., Frank Laboratory of Neutron Physics, Annual Report (2007)

[12] https://wwwinfo.jinr.ru/programs/jinrlib/psd2sas/indexe.html

[13] https://root.cern.ch/ 